\title{
e-Surveillance in Animal Health: use and evaluation of mobile tools
}

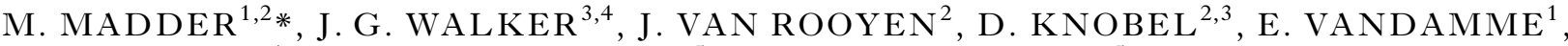 \\ D. BERKVENS ${ }^{1}$, S. O. VANWAMBEKE ${ }^{5}$ and E. M. DE CLERCQ ${ }^{5}$ \\ ${ }^{1}$ Vector Biology Group, Department of Biomedical Sciences, Institute of Tropical Medicine, Nationalestraat 155, \\ B-2000 Antwerp, Belgium \\ ${ }^{2}$ Department of Veterinary Tropical Diseases, Faculty of Veterinary Science, University of Pretoria, Private Bag X04, \\ Onderstepoort, Pretoria, 0110, South Africa \\ ${ }^{3}$ Visiting scientist, KEMRI/CDC Research and Public Health Collaboration, P.O. Box 1578, Kisumu 40100, Kenya \\ ${ }^{4}$ Compton Mentor Fellowship, a program of the Compton Foundation, 101 Montgomery St. Suite 850, San Francisco, \\ CA 94104 \\ ${ }^{5}$ Georges Lemaître Centre for Earth and Climate research, Earth and Life Institute, Université catholique de Louvain, \\ place L. Pasteur 3, B-1348 Louvain-la-Neuve, Belgium
}

(Received 21 February 2012; revised 7 March 2012; accepted 11 March 2012)

\section{SUMMARY}

In the last decade, mobile technology offered new opportunities and challenges in animal health surveillance. It began with the use of basic mobile phones and short message service (SMS) for disease reporting, and the development of smartphones and other mobile tools has expanded the possibilities for data collection. These tools assist in the collection of data as well as geo-referenced mapping of diseases, and mapping, visualization and identification of vectors such as ticks. In this article we share our findings about new technologies in the domain of animal health surveillance, based on several projects using a wide range of mobile tools, each with their specific applicability and limitations. For each of the tools used, a comprehensive overview is given about its applicability, limitations, technical requirements, cost and also the perception of the users. The evaluation of the tools clearly shows the importance of selecting the appropriate tool depending on the envisaged data to be collected. Accessibility, visualization and cost related to data collection differ significantly among the tools tested. This paper can thus be seen as a practical guide to the currently available tools.

Key words: Surveillance, mobile technology, smart phones, SMS, animal health.

\section{INTRODUCTION}

The OIE Terrestrial Animal Health Code defines animal health surveillance as "an essential tool to detect disease or infection, to monitor disease trends, to facilitate the control of disease or infection, to support claims for freedom from disease or infection, to provide data for use in risk analysis, for animal and/or public health purposes, and to substantiate the rationale for sanitary measures” (OIE 2011). Although surveillance itself is defined as a tool for the above purposes, different surveillance systems rely on a variety of datacollection tools which may or may not be usable for purposes other than surveillance. The type of datacollection tool used depends on the type of surveillance being conducted (active or passive), as well as on the role of the people submitting reports. Traditional methods include retrospective and passive surveillance of notifiable diseases, conducted on standard paper forms, which are submitted, consolidated and resubmitted at various levels until the information reaches the OIE. Although such paper-based

* Corresponding author: mmadder@itg.be; +32 3247 6397 collection methods have been used for a long time, paper-based collection methods are not always standardised, are time-consuming and are especially error-sensitive. In addition, with a system such as the traditional one for notifiable disease reports, data may take months to reach a level at which they can be analysed for trends and outbreaks. The consistency and quality of data collection in a format that facilitates analysis is critical to effective surveillance and the main drawbacks of the classical data collection are numerous: inaccuracies in data collection, errors in translation of paper forms to computer databases, duplication of efforts, delay in detection of cases, lack of feedback mechanisms and linkages between levels, delayed response times to events, etc.

Widespread access to the internet has enabled animal disease surveillance systems to improve in terms of speed of submission. However, on the ground and in the field the primary data are still likely to be collected on a paper form, allowing the errors listed above to continue. A rapidly growing alternative to paper-based forms is the use of mobile phones and cellular networks to submit information to a database directly from the field. Such tools offer the advantages of immediate digitization, transmission, 
and aggregation of data, potentially improving speed, cost-effectiveness and accuracy of surveillance (Schuster and Brito, 2011). They have been especially useful in developing countries where access to traditional electricity infrastructure, computers and the internet is limited, but mobile phone access is rapidly growing (World Bank, 2008).

Mobile phone-based surveillance tools may be based solely on the traditional uses of cell phones, including voice calling and text messaging (SMS), or rely on mobile Internet (GPRS/3G) for data submission. Software designed for low-end mobile phones with submission of data via SMS have proven to be extremely useful for human and animal health surveillance in a variety of situations, such as disease reporting after earthquakes (Yang et al. 2009) or reporting of emerging infectious diseases (Qekwana et al. 2010; Robertson et al. 2010). The systems, which use SMS for data submission, such as RapidSMS and FrontlineSMS, run on almost every low-end mobile phone. Personal digital assistants (PDAs), though they do not allow a cellular data submission method, have also been used successfully to digitize data submission, for example in a household health survey (Shirima et al. 2007). In addition to PDAs, smart phones enabled with internet access via a mobile data service such as GPRS, a global positioning system (GPS) and camera allow collection and inexpensive transmission of types of data other than text, and were a milestone for the collection of accurate geo-referenced data (Aanensen et al. 2009).

The popularity of mobile phones and mobile network (GSM)-based services in the entire world and especially in developing countries that lack sufficient fixed-line infrastructure has increased considerably during the last decade. The number of global mobile connections was predicted to reach six billion by the end of 2011, with 500 million lines, equivalent to about $50 \%$ of the population, in Africa (Blycroft, 2008). The local and regional investments of telecom companies lie at the basis of the success of mobile communication. This system is certainly more cost effective compared to fixed-line telephony, both for network operators and for consumers. Due in some cases to competition between service providers, mobile phone handsets, connection fees, and cost per minute or per message have become affordable to most people in the developing world. The prepaid option is extremely popular for lowincome users as the user pays only when they use the service; very popular are affordable low-end mobile phones that offer basic telephony such as voice and text messaging, being purchased new for as little as about $\$ 10$.

This paper does not aim to give a complete overview of all e-surveillance tools available (such overview has been compiled by MobileActive.org, 2010), but rather to share our findings on some tools that we have been exploring in the past few years.
These tools were chosen especially for their characteristics and applicability in the different domains of research taking into account the availability of mobile devices. Low-end mobile phones in combination with the open source software JavaRosa and RapidSMS, as well as smartphones running EpiSurveyor were successfully used in a syndromic livestock disease surveillance system in Kenya. PDAs equipped with SurveyToGo were tested to get base-line data about the ticks and tick-borne disease importance in a rural community in South Africa at the wildlife-livestock interface. Smart phones with GPS in combination with the software EpiCollect were used to monitor the recently introduced cattle tick Rhipicephalus microplus in West Africa. In the latter project other electronic tools were introduced to facilitate tick identification under field conditions, including mobile USB microscopes and an electronic and interactive Bayesian key.

For each of the tools used, their requirements, characteristics, advantages and drawbacks are given.

\section{CASE STUDIES}

\section{Monitoring invasive tick species in Benin using EpiCollect, USB microscopes and interactive, electronic Bayesian identification keys}

Introduction. Changing climate, globalization, animal movements and livestock improvement programmes, on their own or in combination, have resulted in changing vector distribution patterns. Animal movements that are due to natural migration (i.e. birds carrying immature tick stages) or are anthropogenically controlled (transport of domestic and wild animals) significantly increase the risk of vector introduction. The introduction of Rhipicephalus microplus in West Africa is considered the most recent and potentially most important case of vector import (Madder et al. 2007, 2011, 2012). Because of the suitable habitat and large livestock presence, the impact on livestock production and health might be significant. Several activities and projects in the region (Weca'Tick and TickRisk) focus on the extent of spread of this vector and its resistance to acaricides. TickRisk, funded by the Belgian Federal Science Policy Office, aims to develop habitat suitability maps for R. microplus in West Africa and to assess the usefulness and feasibility of using 'new' technologies such as smart phones, mobile devices and interactive identification tools to collect information for incorporation in web-mapping tools to be used by local stakeholders as well as researchers. The challenges of this project were to overcome the difficulties of tick identification in the field or in the absence of an entomological laboratory, the collection of field data by electronic means and the geo-mapping of vector presence. Three tools were identified and tested: EpiCollect for the electronic data collection, transfer 
and mapping, the Veho USB microscope for field visualisation of ticks and iSpot as an interactive Bayesian identification key.

Material and Methods. EpiCollect (www.epicollect. net) is a web freeware application (www.epicollect. net) developed at Imperial College London and funded by The Wellcome Trust, and runs on both Android- and Mac iOS-supported devices. The application allows multiple surveyors to collect and submit data by smart phones, including GPS data and images, to an online database. These data and previously collected data can be displayed, analysed, filtered and mapped using Google Maps, both on smart phones and PCs (Aanensen et al. 2009). A google account is required for the creation of a project and the related questionnaire. The data are not password protected, and can be viewed or downloaded if the URL of the project is known. The application has a low learning curve and a complete project can be set-up in a few hours, including the development of the website, form to collect data, installation of the mobile application and first transmission of test results. On the entry page of EpiCollect a new project can be created by just entering a project name. This unique name will allow all surveyors to download the forms created by the author of the project. For the TickRisk project basic information about the location, import or export of animals, acaricide application, and presence of ticks is asked, without the necessity to add information about the identification of the ticks collected at that location. That information is entered afterwards via the smart phones or directly into the online database by the author of the database. During data collection/ entry, the GPS location is automatically recorded in the forms and if necessary, a photo can be added to each survey. Entered records, both locally and externally recorded by other surveyors, can be viewed on the smart phone and on the website. The latter offers the option of displaying up to four variables in a chart and filtering data by variables or by entry time, these options are ideal for monitoring of data in realtime during ongoing fieldwork. Via the website, communication between all online users is available via instant messaging provided by Google Talk.

The identification of ticks, especially ticks of the genus Rhipicephalus (Boophilus) is cumbersome, mainly because of their small size and limited differentiating morphological characteristics. A powerful stereo-microscope is essential, which is often not available in many laboratories in developed countries and certainly in low-income countries. Alternatives for accurate identification are limited. Veho USB microscopes however, present an excellent alternative: a camera that offers high resolution images of $2 \mathrm{MB}$ at high magnification (up to $400 \times$ ) and that allows visualization of the smallest morphological details necessary for identification of Rhipicephalus (Boophilus) species.

The installation of the Veho software and set-up and use of the microscope is extremely easy and does not require any prior knowledge. However, a netbook, laptop or PC is required because no applications or drivers are available to connect the USB microscope to a smartphone or tablet PC.

The identification of most arthropod species requires a lot of experience and knowledge and, if present, it is normally related to species of a specific geographic area of interest. The recognition of 'exotic' species is not always obvious and the use of dichotomous keys might not pick them up. The use of iSpot, an interactive electronic key that uses Bayesian analysis to calculate probabilities of correct identification based on the input of specific information in the key, offers a better tool to identify arthropods or any other organisms for which a key has been developed (see the iSpot.org.uk site for examples). The development of such keys does not require any programming skills. The system is developed in such a way that the key is automatically generated from information provided in a spreadsheet (.xls) and a folder containing the related photo material.

In the TickRisk project, six local teams from the Department of Animal Production, each located in one of the six departments of the country, were equipped with an Android-based cellphone (LG Optimus $\mathrm{Me}(\mathrm{P} 350), 100 €)$ with EpiCollect installed, a USB microscope (Veho VMS-004D $400 \times, 80 €$ ) and a netbook (Acer, $250 €$ ), that would allow them to collect and transmit additional data apart from the foreseen and budgeted sampling places, and also identify ticks. Because no specific budget was available for the additional collections, no specific expectations were set. Especially for the tick identification and the use of iSpot, but also for the use of EpiCollect and the USB microscope, a training session of two days was organized for the six teams. Documents describing in detail the protocol to be used were drafted and distributed to the participants of the workshop.

Besides collections of local teams, four tick collection missions of 10 days each were organized to collect ticks in about 130 farms dispersed in the six departments of Benin, and this by a team of international and local scientists.

Results/Discussion. Setting up the entire surveillance system, including the EpiCollect website, testing the USB microscope and developing the iSpot identification key for ticks belonging to the genus Rhipicephalus (Boophilus) was extremely fast. The most time-consuming part (1 week) was to create the iSpot key (See Fig. 1), the rest was done in less than a day. 


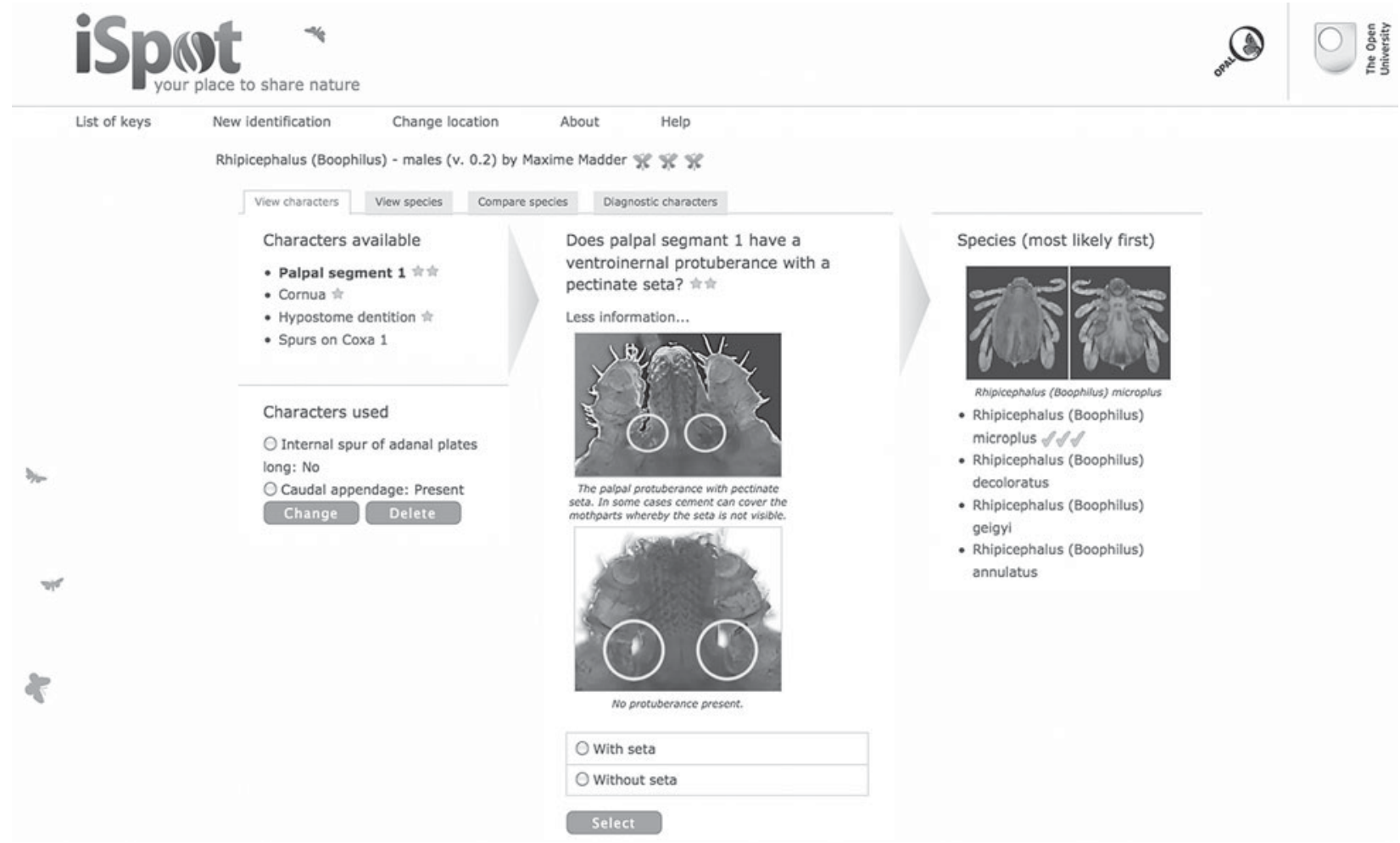

Fig. 1. Screenshot of the iSpot keys for males of Rhipicephalus (Boophilus) ticks. On the left the different characters are listed, both characters available and used. The central panel presents the character being evaluated with all necessary visual aids. At the right side of the window the probability is given of all species of the genus, the highest in this case is for R. microplus.

During the tick surveillance and collection missions in Benin, the use of EpiCollect was positively evaluated. The software was used and tested by the authors and by local stakeholders. Getting acquainted with its use and functionality was easy and no special training was needed, except for a brief demonstration during the surveillance. For transmission of the data we opted for a once-off transmission to be able to calculate the total cost of data transfer. During each mission, roughly 40 farms were visited and a similar number of records were saved and uploaded to the EpiCollect server. It should be mentioned however that data transfer requires internet access from the cellphone or a wireless connection to an internet network (directly via Wifi or indirectly via a connection to an internetenabled computer). Internet access via the cellphone network is not always possible in resource-poor settings but EpiCollect is able to store data locally on the phone until connection with the server is possible. The collected data were uploaded to the server through a Wifi-network. In order to obtain a cost estimate for upload using a wireless cell phone connection, a limited number of tests-uploads was performed, yielding a mean upload cost of $0.03 €$ per 10 records.

The power of EpiCollect is its real-time and online mapping facility. Once data are synchronized, they are mapped and accessible via the project website. All stakeholders are able to follow and view all the details of each collection point. Especially for emerging vectors or diseases, this facility offers great advantages for information dissemination and rapid intervention. On the project website, data can be filtered and represented in graphs to give a quick overview of the different variables recorded (see Fig. 2).

After sample and data collection, the ticks were identified. To get an initial idea of the presence or absence of the newly introduced tick species in the different locations sampled, a sub-sample was identified in the field by using the USB microscope. For practical reasons, all other ticks were identified in the laboratory of the Vector Biology unit at the Institute of Tropical Medicine, Antwerp in Belgium.

Within the first four months after the transfer of the hardware to the local stakeholders, a limited number of data points were covered by the local teams, most probably because of lack of resources to do field collections or difficulties to get access to the hardware. From the limited data transferred and available at the EpiCollect website, it was clear that some local teams were exploring the tools and collecting and transmitting data. During the next partner meeting of the project, an evaluation will be made of the usefulness of the electronic tools. In general, surveillance of vectors has become more efficient and accessible in low-resource settings by using mobile tools. 


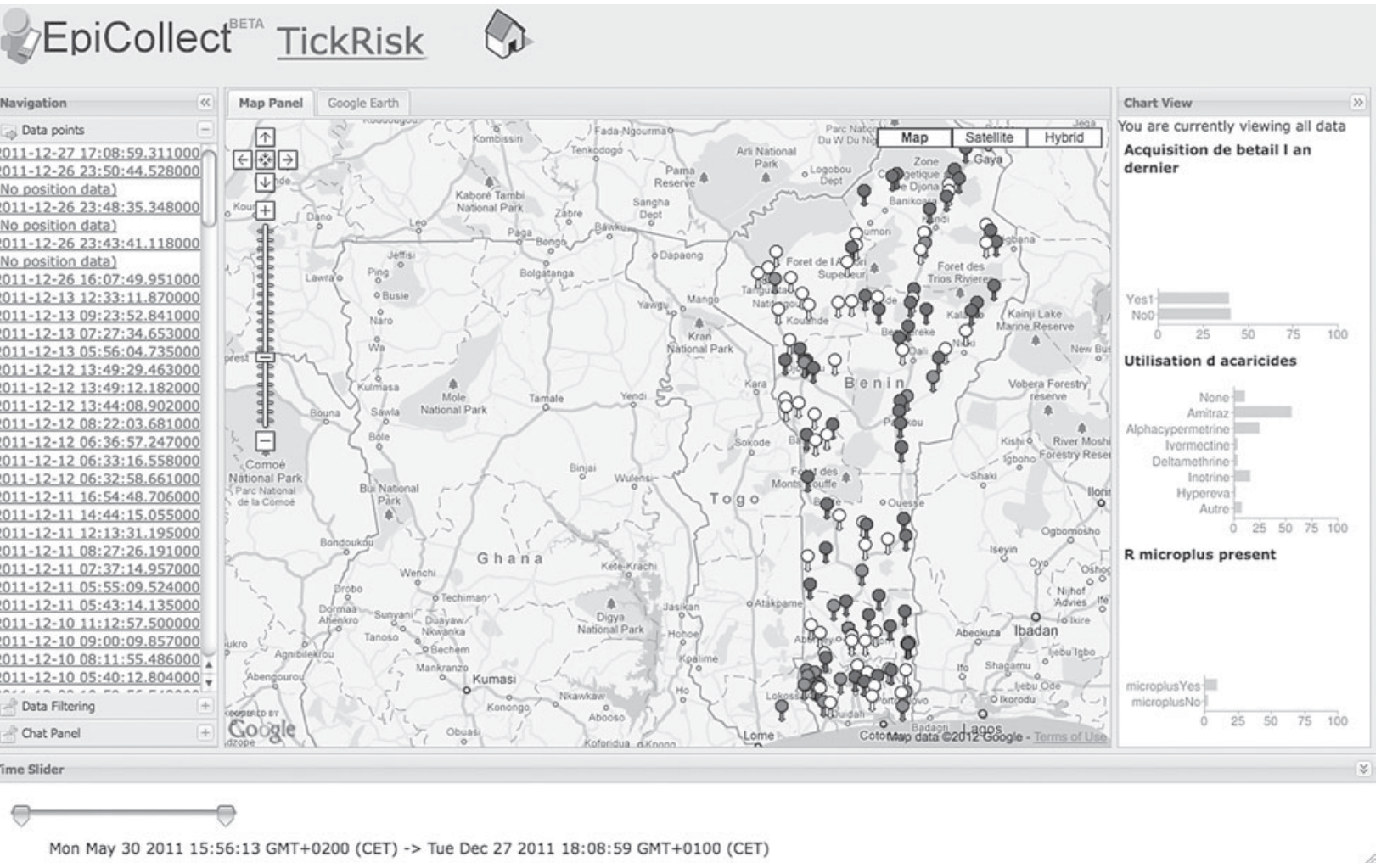

Fig. 2. Screenshot of the EpiCollect data page of the TickRisk project. The left window shows the data entries, filtering and chat options. The central window presents the geographical distribution of the data points. Some of the variables collected by EpiCollect can be charted to give a first impression of the data, presented in the right window.

\section{e-Surveillance of ticks and tick-borne diseases at the wildlife-livestock-human interface in Mpumalanga province, South Africa using SurveyToGo}

Introduction. The collection of base-line data for the presence, impact and control of ticks and tick-borne diseases in the cattle of communal farmers at the human-livestock-wildlife interface required a different approach compared to the monitoring of R. microplus distribution as described above. This study was conducted in the Mnisi community bordering the Kruger National Park in the Bushbuckridge district of the Mpumalanga province of South Africa. This type of study aims to collect detailed quantitative information from communal farmers through interviews performed at the weekly gatherings during the compulsory dipping of cattle. In contrast to vector monitoring, these surveys probe for a much larger amount of data, not always in a linear way, but guided by the events that took place. In short, the number of questions asked of a farmer depends on the answers he gave to previous questions: i.e. if one would get the response that no acaricides were used during the preceding 12 months, it would not be necessary to ask the farmer which products he used or if the products were effective. When using the 'standard' paper-based forms, the surveyor would decide which questions to ask, or which questions to skip. This can become confusing and impractical if large questionnaires are deployed.
After the process of data collection, these data would then be transferred from paper to an electronic database, a time-consuming exercise that is errorsensitive. Here again mobile tools and related software offer excellent alternatives in that large datasets could be exported in ready-to-use digital formats for statistical analyses.

Materials and methods. For this study SurveyToGo (STG) (www.dooblo.net) was used. It is mobile data collection software that enables users to create, conduct and manage electronic mobile surveys on a Windows laptop, and deploy them on an Android or Windows Mobile PDA or mobile phone. It is provided as a hosted cloud service and is not freeware although costs related to performed surveys for academic purposes are limited to a maximum of $0 \cdot 20 €$ per survey, independent of the number of questions in the survey. Apart from the ability for GPS location and picture capturing, and video and voice recording, it is highly appreciated for its large number (13) of question types and especially for its piping, branching, skipping, validating, looping and randomizing options. These options allow the survey designer to incorporate quality control of numeric data and guidance of the flow of the survey: questions to be asked or skipped. Guidance can be achieved by composing rules: (1) entry rules control whether the question is displayed or not, (2) validation rules 


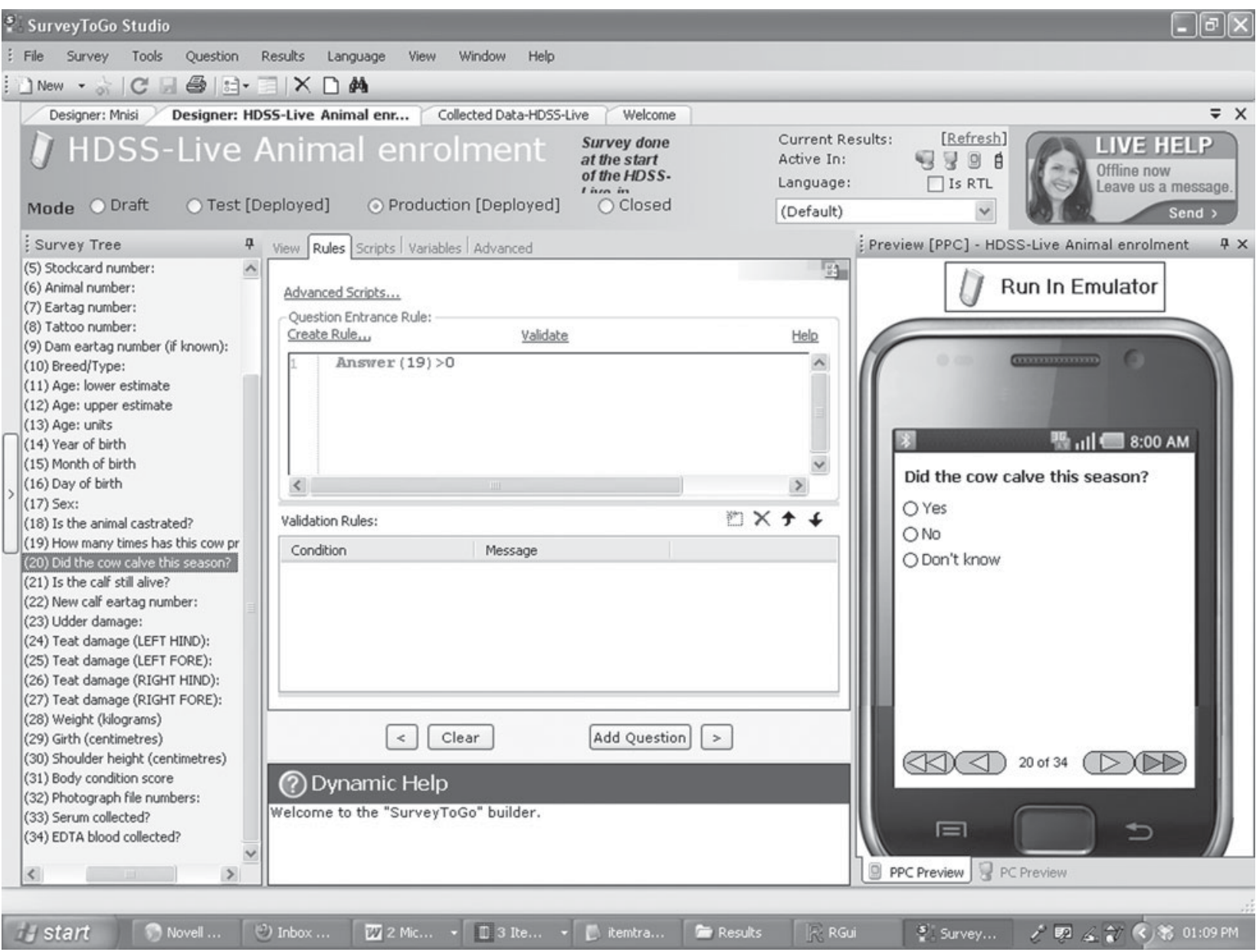

Fig. 3. Screenshot of SurveyToGo. The left window shows the different questions. In the middle window the rules of the highlighted questions are presented (jump rules in this case) and in the right window the emulator shows how the question appears on the device.

control whether the question is valid or not and (3) jump rules control the action to perform in response to the question answer.

All data generated as well as the associated questionnaires were stored on a secure server hosted by Dooblo and is protected by different levels of password protection as managed by the project administrator(s).

In this study we opted for iPaq110 units (120€) because of their large and bright screens and powerful batteries. These devices are not equipped with a camera or a GPS receiver. The need for power backup was foreseen and Solar and PowerGorilla systems were set up, though they turned out not to have been necessary for the success of the study.

Results/Discussion. The initial survey was prepared as a text file and consisted of 151 questions. The questions were defined after focus group discussions were organized at the study area. Once the survey questions were identified and approved, the STG survey was designed, mostly by copy and paste from the text file (See Fig. 3). The challenge consisted of using the appropriate STG "rules" (defined above) to make the survey more user-friendly than the text file or paper-based survey to avoid errors and save precious time performing surveys during the field missions. Once the STG survey was build, it was tested and fine-tuned in silico. All further steps were performed in the field. As a first step the four survey teams, consisting of an interpreter and a surveyor, were identified and the interpreters were trained during a two day training course. The first part of the training focused on the explanation of technical terms, the questions, their meaning and the information they probe for with the help of local experts fluent in English and Shangaan. The basics of interview techniques were explained with the emphasis on the danger in offering answers to the farmers during questioning, instead of rather trying to explain what information we were looking for. No technical training for the use of STG or mobile devices was given to the interpreters but only to the surveyors not previously exposed to the methodology during separate training sessions. The second phase of training consisted of the team being deployed in the field at two dip tanks outside of the study area. This allowed us to identify the last inaccuracies or adjust questions types or variables, test local data transfer possibilities and finally download the 
corrected survey onto the mobile devices. This exercise further highlighted the importance of testing for internet connectivity under field conditions before the onset of the survey. Internet access in the field is required for the purpose of sychronising questionnaires and results with the Dooblo server for either the safe storage of data or if new or existing questionnaires are deployed/adapted remotely.

During the next ten days, the survey teams performed 144 surveys during the early morning cattle dipping sessions, on average four surveys per team per dipping session. The surveys were conducted between $5 \mathrm{am}$ and approximately $10 \mathrm{am}$, the time during which the farmers normally gather at the dip tanks for compulsory dipping and inspection of their cattle, and lasted on average 50 minutes per questionnaire. Synchronization of the data was done on a daily basis, but because non-mobile connected PDAs were used, Wifi or direct connection to a PC was necessary. A Wifi connection was available at the South African Wildlife College near the study area. Synchronization of all the devices only required five minutes, less than half a minute per device, but this was certainly related to the connection speed available at the College. However, during testing it was found that the updating and adapting of a questionnaire in the field on the computer using a weak GPRS internet connection could significantly increase the synchronization time than when using broadband or when merely synchronizing the mobile units.

Although a paper-based survey was not run in parallel, previous experiences with the traditional type of surveys demonstrated the user-friendliness of STG and in general of an electronic survey tool. Guidance based on the built-in rules of questions to be addressed is one of the most appreciated advantages experienced during the survey. It allows the surveyor to focus on the interview and the answers given by the respondents without the need to look for the next question. This saves precious time and allows for a more fluent conversation with the respondent. It further prevents the surveyor from losing focus and enables the surveyor to perform more surveys in the limited time available while the farmers are waiting for their animals to be dipped. Another big advantage of this method is that no manual, labour-intensive and error- sensitive data processing from a paper to a digital format was necessary after the survey other than standard data cleaning.

The challenge we faced in this case study was to translate an existing paper-based survey to the electronic STG version and then use the appropriate tools to make the survey as efficient as possible. Compared to EpiCollect this software tool has a steeper learning curve but offers much more possibilities for large surveys. As the mapping facility offered by EpiCollect was not a requirement in this study, STG seemed much more appropriate and powerful. However, the latest version of STG do have some interactive mapping functionality available.

\section{Syndromic livestock disease surveillance in Kenya using RapidSMS, FavaRosa, and EpiSurveyor}

Introduction. Livestock production is economically important in Kenya with livestock production contributing $12 \%$ of GDP (Anon, 2005). Although some large-scale dairy farms exist, the livestock sector is predominantly made up of small-scale subsistence farmers in rural areas. In the rural division of Asembo in Nyanza province in Kenya, 89\% of the population own domestic animals. The livestock density in this province is the second highest in the country, with 137.96 cattle per square kilometer (OIE, 2009). Despite the prominence of livestock, animal owners have limited access to healthcare for their animals, both logistically and financially, except during occasional government-led vaccination campaigns. Kenya is ranked $61^{\text {st }}$ overall out of 89 countries by the ratio of veterinary personnel to livestock (OIE, 2009). In this context, we developed a mobile phone-based syndromic disease surveillance system which relies on reports of illness by animal owners, this triggers a response by a team of animal health technicians who respond to each case and collect clinical signs information and samples for laboratory diagnosis. The goals of the system were to establish a baseline knowledge of disease in the area which can be used to help detect outbreaks early, as well as to provide local people with access to veterinary personnel that can answer questions and offer care for their livestock.

Material and methods. A combination of mobile data collection tools was used in this system because the system consists of two separate surveillance levels. The first level consists of the initial report of illness in an animal, with information on the location, species of animal and syndrome exhibited. One to two people from each of the 33 villages in the area were identified to act as reporters and submit reports when an animal in their village became sick with a relevant syndrome (sudden death, abortion, live birth or nervous signs). This information was submitted via SMS from a basic (Java-enabled) mobile phone using the software JavaRosa on the handset and submissions were received by the software RapidSMS on a central server. The second level is the data that were collected by members of a six-person team of trained animal health technicians that responded to each reported case. These data were collected on a smart phone running the Android operating system and the software EpiSurveyor and submitted over the mobile internet network to a database accessible on the internet. 


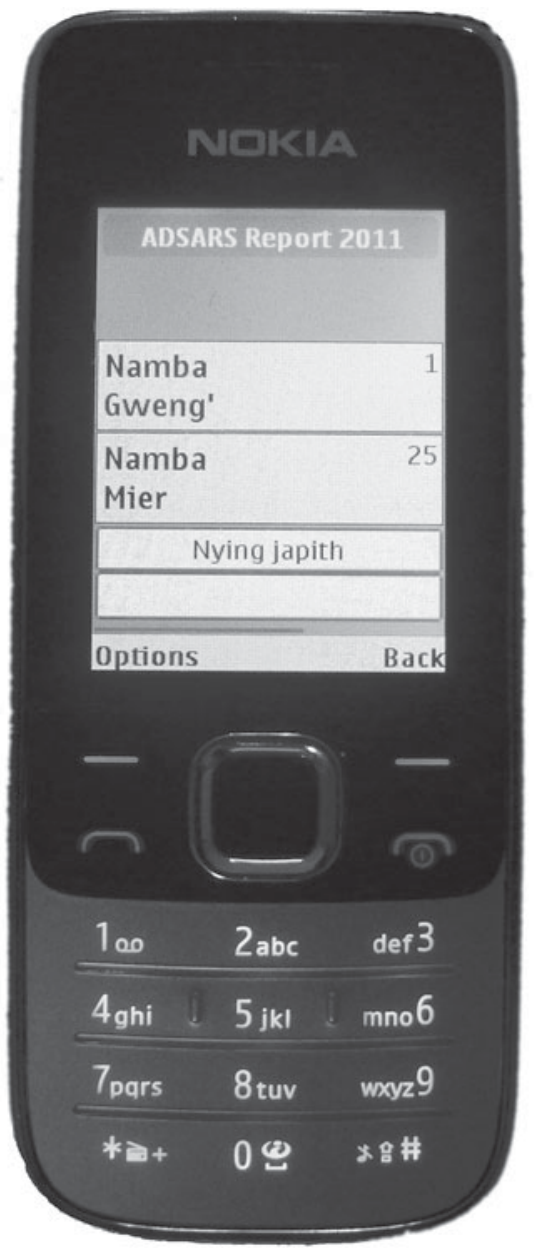

Fig. 4. JavaRosa on a basic mobile phone.

One of the primary limitations of data submission by SMS from a simple low-end phone is the need for the text message to be formatted in a particular way in order to be parsed by the software program to which it is submitted. Although RapidSMS can receive and process a basic SMS which begins with a simple key word, we wanted to use the functionality provided by the add-on XForms app, which parses a strictly structured SMS into automatically-filled fields in a database. Our end users were animal health reporters who were selected and hired based on their knowledge of and influence in the community rather than their expertise in phone use. Although all of them owned or had access to a mobile phone, these users would have had trouble consistently composing messages with a rigid structure, including the use of spaces and ' + ' to mark new fields, which is required by the XForms app. We avoided this problem by providing Java-enabled phones, which are slightly more expensive than the most basic phones and can run basic software programs. The handsets we used were Nokia 2730c, which at the time was available in Kenya for $6,999 \mathrm{KES}(63 €)$. This phone is $3 \mathrm{G}$ internet enabled, has a 2.0 megapixel camera, Bluetooth and a microSD card slot. We installed software called JavaRosa on each phone (See Fig. 3).
The software allows the use of a graphical user interface to fill out a form with several fields, including free entry fields and check boxes for the type of animal and syndrome exhibited, and then to submit the contents of that form in a structured way to RapidSMS, where the information is parsed into a database and a message notifying the response team of the submission is automatically and instantly forwarded.

JavaRosa is an open-source mobile data collection tool that forms the basis for a number of other existing tools, such as CommCare, a data collection tool for community health workers. It is primarily developed by the OpenROSA Consortium and the software consultancy company Dimagi. JavaRosa runs on any Java-enabled phone (See Fig. 4), but works best on phones that have a heap size (memory) of $2 \mathrm{mb}$ or more. JavaRosa displays XML forms that are created using the XForms guidelines; some programs are available online which allow the creation of the forms by use of a user-friendly interface, or the code may be written from scratch. JavaRosa works quite well on these phones although it takes about 15 seconds for the program to load and open. The microSD card that came with the phone is useful for transferring the software from the computer, but it can also be transferred by Bluetooth or downloaded directly to the phone from the internet. The software is easy to set up and install with a basic knowledge of file transfer on computers and mobile phones, however, for editing the XML forms to allow submission by SMS a basic understanding of XML was helpful. The JavaRosa community is very helpful and instructions are available on their website and in the Google group archive (www.dimagi.com/ javarosa/, http://groups.google.com/group/javarosadevelopers/topics).

RapidSMS is free and open-source software designed to manage data submitted by SMS. The software runs best on Linux and requires some technical or programming expertise to implement. The RapidSMS server is a computer running Linux that has been connected to a GSM modem. The modem we are using is a Huawei E160 modem sold by Safaricom, this is one of the older generations of Safaricom modems, but some of the newer modems are not known to be compatible with RapidSMS. A new modem is currently available from Safaricom for 1,999 KES (18 €) RapidSMS can also present information on the Internet as soon as it is received if the server has access to a public IP address.

EpiSurveyor is a free (within limits) closed-source software program for data collection that is designed to be easy and quick to implement by users with limited technical expertise. Their goal is to be "the Gmail of data collection.” EpiSurveyor is developed by the software company DataDyne (www.datadyne. org). It runs on a wide range of java-enabled phones, the Android operating system as well as on iPhone 


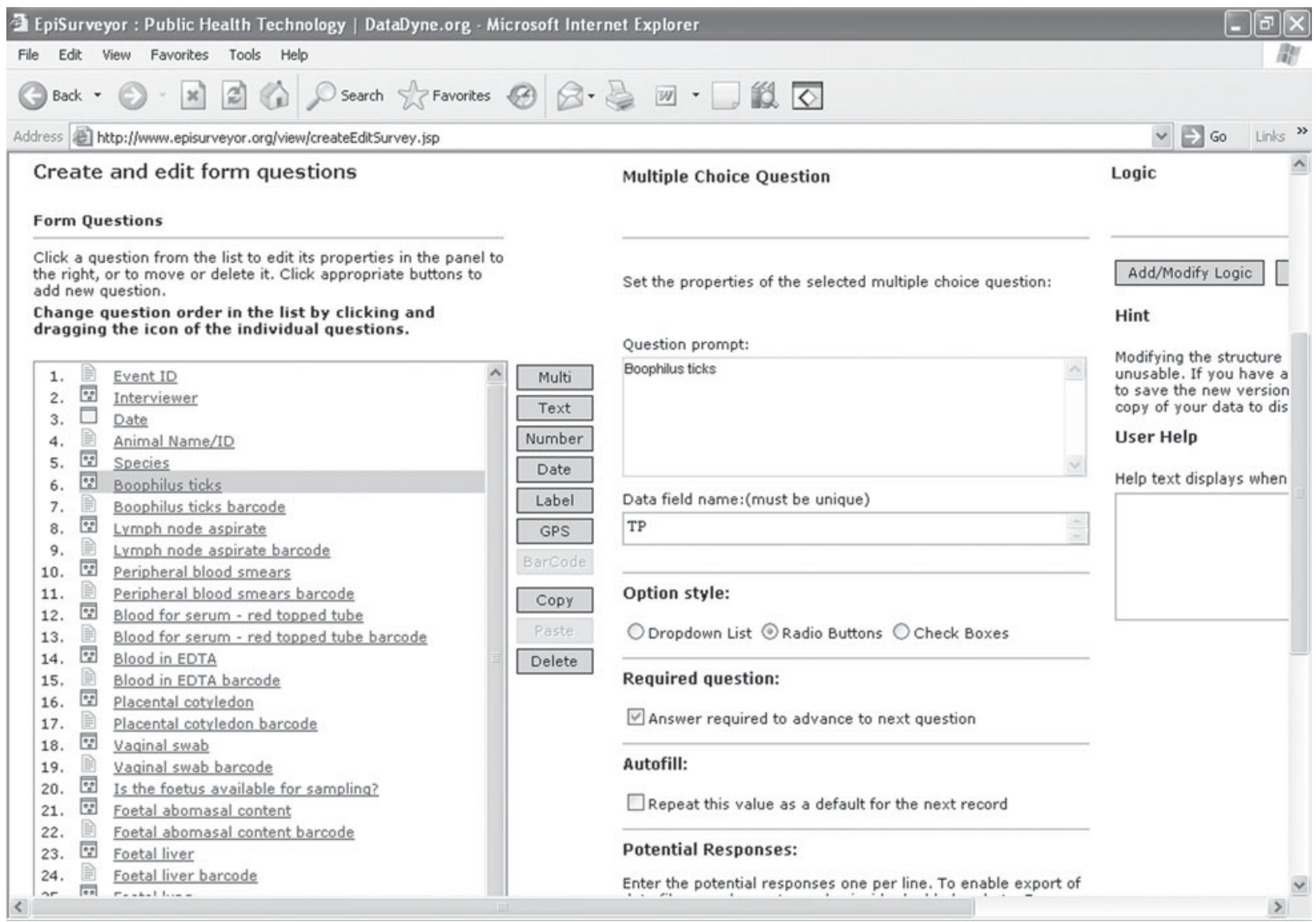

Fig. 5. Screenshot of EpiSurveyor. The left window lists the questions of the survey whereas the middle window presents the details of the highlighted question.

although it requires iOS 4.0 or later. The EpiSurveyor iPhone app is not freeware however but costs less than $2 €$.

We are running EpiSurveyor on an Android smartphone, the Sony Ericsson XPERIA X10 Mini Pro. This is a relatively inexpensive smartphone with a 5.0 megapixel camera, a slide out QWERTY keyboard, touchscreen, $3 \mathrm{G}$ Internet access, GPS, Bluetooth, and a microSD card. This phone is available for sale in Kenya at 23,999 KES (215 €) or an international version can be purchased on Amazon.com.

Although EpiSurveyor runs on cheaper and lower end phones, due to the large amount of information that we would be collecting using this software it made sense to use a smartphone with a relatively large touchscreen and a physical keyboard for ease of data entry. Installation of the software on the phone is simple, and just requires use of the phone's web browser to navigate to www.episurveyor.org/m and selection of the appropriate version. The software automatically downloads and installs.

In order to create forms for data submission, it is necessary to create an account at www.episurveyor. org. Forms are easy to create online using a simple interface (See Fig. 5). There are a number of aspects of EpiSurveyor that are quite convenient - an account can be set up within minutes, forms can be created without technical expertise, and they have an online emulator so that you can test what a form will look like without a phone. However, there are also a number of inconvenient aspects of EpiSurveyor. First, the creation of a form using their online interface can be quite frustrating when using a slow internet connection. If they had the option to upload forms in the standard XML format used by many other programs then the opportunity to work on the forms offline would be available to those with a bit of technical experience. Second, the free account sets limits on the number of forms each user can own, the number of records that can be stored in each form, and the number of transfers of data per year, paid accounts are designed for large organizations and cost either $3800 €$ or $7200 €$ per year (DataDyne Group LLC 2011). Another inconvenience of EpiSurveyor relates to the hosting of data. The data in a free account are all stored on the EpiSurveyor servers, and for some organizations that collect very sensitive data, for example on human health, this may not be advisable. On the other hand, the system uses secure (HTTPS) transfer protocols to submit data and EpiSurveyor takes responsibility for making sure the data are always secure, backed up, and available online. Data are only visible to the user who created 
Table 1. Overview of the tools used and their specifications

\begin{tabular}{|c|c|c|c|c|c|}
\hline Tool & What is it? & $\begin{array}{l}\text { Technical } \\
\text { expertise }\end{array}$ & $\begin{array}{l}\text { Hardware } \\
\text { required }\end{array}$ & Cost & website \\
\hline Epicollect & $\begin{array}{l}\text { Data collection for } \\
\text { Android or iOS, data } \\
\text { submitted directly to, } \\
\text { hosted and accessible } \\
\text { on internet, including } \\
\text { GPS and photo }\end{array}$ & Low & $\begin{array}{l}\text { Computer with } \\
\text { internet access, } \\
\text { Android } \\
\text { smartphone or } \\
\text { iPhone }\end{array}$ & $\begin{array}{l}\text { Software free to } \\
\text { use, phones } \\
100 € \text { and up }\end{array}$ & www.epicollect.net \\
\hline Veho USB & $\begin{array}{l}\text { A USB powered } \\
\text { microscope providing } \\
\text { up to } 400 \times \\
\text { magnification }\end{array}$ & Low & $\begin{array}{l}\text { Computer, } \\
\text { microscope }\end{array}$ & $\begin{array}{l}\text { Microscope } \\
80 €\end{array}$ & $\begin{array}{l}\text { http://www.veho-uk.com/ } \\
\text { main/shop.aspx? } \\
\text { category=usbmicroscope }\end{array}$ \\
\hline iSpot & $\begin{array}{l}\text { Web accessible } \\
\text { identification keys }\end{array}$ & Low & $\begin{array}{l}\text { Mobile phone or } \\
\text { computer }\end{array}$ & free & Ispot.org.uk \\
\hline JavaRosa & $\begin{array}{l}\text { Open-source software } \\
\text { that runs on } \\
\text { java-enabled phones } \\
\text { and allows data } \\
\text { submission by SMS } \\
\text { or GPRS }\end{array}$ & Moderate & $\begin{array}{l}\text { Java-enabled } \\
\text { phone }\end{array}$ & $\begin{array}{l}\text { Software free, } \\
\text { phones } 40 € \\
\text { and up }\end{array}$ & www.dimagi.com/javarosa \\
\hline RapidSMS & $\begin{array}{l}\text { Open-source software } \\
\text { that receives and } \\
\text { processes data sent by } \\
\text { SMS }\end{array}$ & High & $\begin{array}{l}\text { GSM modem, } \\
\text { Linux computer }\end{array}$ & Modem $20 €$ & www.rapidsms.org \\
\hline EpiSurveyor & $\begin{array}{l}\text { Survey design, data } \\
\text { collection on } \\
\text { java-enabled or } \\
\text { Android phone, and } \\
\text { data hosting online }\end{array}$ & Low & $\begin{array}{l}\text { Java-enabled } \\
\text { phone, Android } \\
\text { smartphone or } \\
\text { iPhone }\end{array}$ & $\begin{array}{l}\text { Phones } 40 € \\
\text { and up }\end{array}$ & www.episurveyor.org \\
\hline SurveyToGo & $\begin{array}{l}\text { Survey design, data } \\
\text { collection on PDA, } \\
\text { and data hosting } \\
\text { online }\end{array}$ & Low & $\begin{array}{l}\text { Windows Mobile } \\
\text { or Android } \\
\text { PDA or phone }\end{array}$ & $\begin{array}{l}\text { PDA } 120 € \text { and } \\
0 \cdot 20 € \text { per } \\
\text { survey }\end{array}$ & $\begin{array}{l}\text { http://www.dooblo.net/stgi/ } \\
\text { surveytogo.aspx }\end{array}$ \\
\hline
\end{tabular}

the forms (the owner) or those that the owner has specifically shared access with. Data are stored online, and with a free account it is not possible to host data on own servers or set up an API to download the data automatically to another server. Instead, we have decided to download all the records manually on a weekly basis and store them offline in an SQL database. This is quite time intensive especially with a slow internet connection. The final inconvenience of EpiSurveyor is that it is not possible to create a relational database. This means that we have several different forms that need to be filled out and the unique identifiers for particular cases need to be entered separately into each form. This leaves a lot of opportunity for errors in entry and means that running regular data-cleaning checks on our offline database is crucial for catching errors when they can be still be corrected easily.

Results/Discussion. Challenges to implementation of a livestock disease surveillance system include inadequate road network and electricity infrastructure. Data submission over mobile networks is reasonable because of the extensive coverage by mobile network providers in Kenya. However, costs and accessibility vary by the method of data submission, SMS versus mobile internet protocols such as GPRS or EDGE.

Data submission by SMS is expensive for the amount of data you can submit. In Kenya, the price of an SMS on Safaricom is only 1 KES (approximately $0.009 €$ ), but each submission is limited to 160 characters. SMS is a reliable submission system that will send even in an area with very limited network coverage. SMS submission may be the best option when users only have simple phones, there is inconsistent network coverage, and where the data to be collected are limited.

Data submission by mobile internet protocols, such as with the software EpiSurveyor, is inexpensive and a large amount of data can be transferred very quickly. However, it requires a different kind of network coverage than that required for voice calls or SMS. Although this network coverage is rapidly growing, it is still limited in Kenya compared to the standard network. In addition, it requires a phone that can access the cellular internet networks, which the most basic and inexpensive phones cannot do. Mobile internet may be the best option in cases where a large amount of data needs to be transferred at once, where users have experience using smartphones or feature phones and access to such phones, 


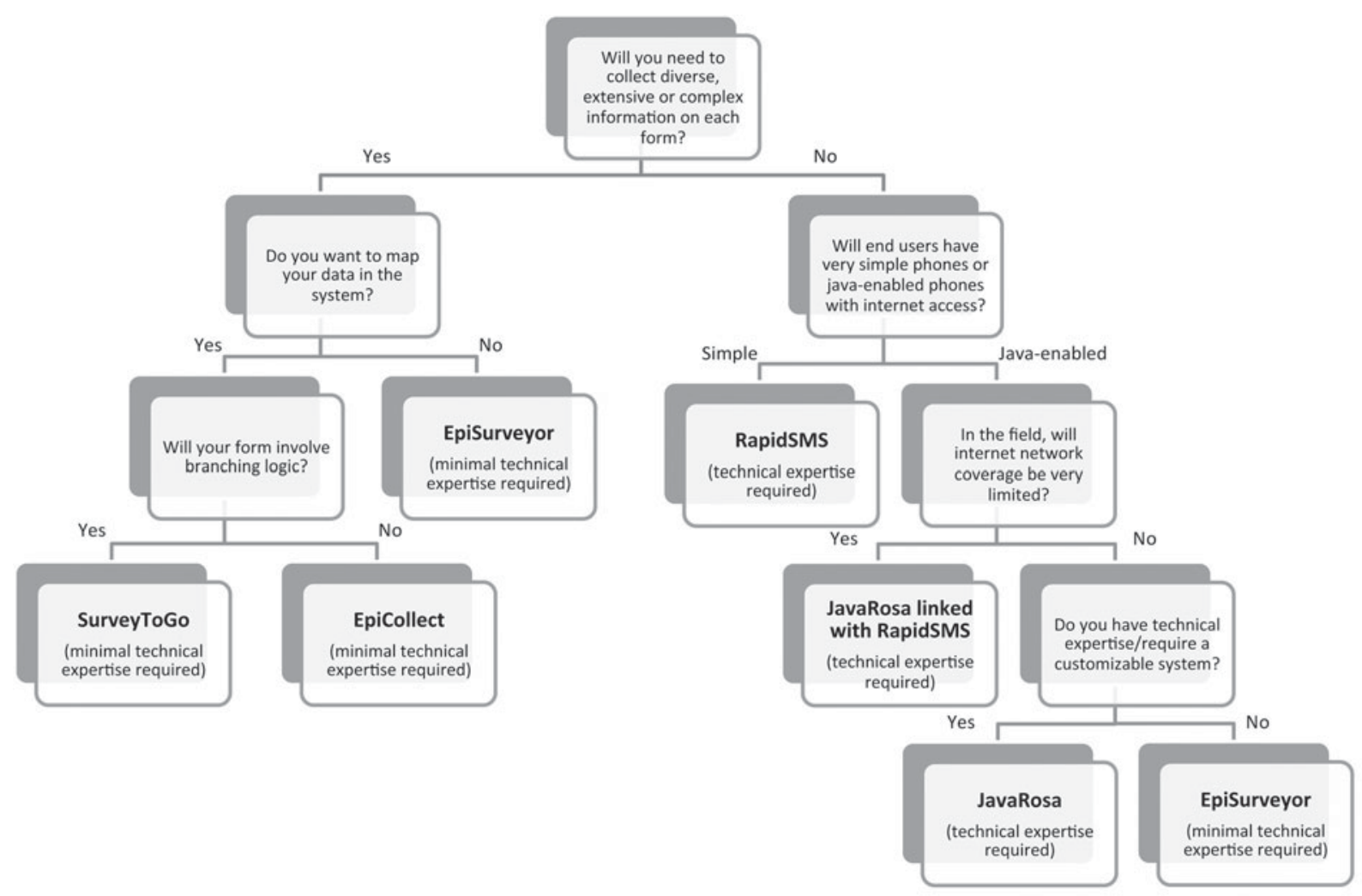

Fig. 6. Decision tree for choosing between the tools presented in this paper. A more extensive list of available tools and their characteristics is kept up-to-date in a spreadsheet at http://www.mobileactive.org/go-to-mobile-data-collectionresources.

and where the mobile internet network coverage is good.

Electronic transmission over mobile networks could allow for more timely transmission of animal disease surveillance data and trigger alerts of outbreaks of zoonotic diseases and economically important diseases of livestock early enough in the outbreak for preventive measures to be taken. By conducting syndromic surveillance, it is possible to detect a wide range of diseases other than those that are currently notifiable, such as new or emerging diseases.

After only one or two short training periods, the users of each mobile data submission tool were able to use the programs to submit information. For any mobile data collection, training in the use of the software and hardware is essential to maximize participation. It cannot be assumed that users will understand how to use the tool without direct training.

\section{GENERAL DISCUSSION}

The use of mobile technology for surveillance in animal health and more broadly for development projects has been expanding rapidly in the past few years. Since much of the software and technology is new, many challenges to implementation are being faced. The rapid development of new and better technology may also leave those interested in implementing mobile technology struggling to keep up. Groups such as HumanIT at Karlstad University in Sweden (http://www.kau.se/en/humanit) have begun to focus on the use of mobile technology for development and host a bi-annual conference where research in the field is shared. Despite the rapid growth of the field, there has not been much published in the peer-reviewed literature, though many reports can be found online, such as at mobileactive.org. The barriers to publication of articles on the use of mobile technology include the interdisciplinary nature of the papers, as well as the need to present failures as well as successes in order to contribute to further research most effectively.

The mobile data collection options available are expanding and improving rapidly. In the case of open-source systems such as JavaRosa, the community building the software is large and many new features are introduced each year. In this paper, we have described the use of several different systems that were used in animal health-related surveillance. Table 1 describes the basic information about each of the systems described above, but these characteristics may change so the websites are provided for future reference. The choice of mobile system to use depends on the needs of an individual project and the resources available to implement it. A table presenting basics on a wider range of mobile tools is 
compiled here http://mobileactive.org/go-to-mobiledata-collection-resources. In addition, a decision tree is provided to assist the reader in choosing the appropriate tool, based on his or her technical expertise, phone characteristics, type of data to be collected and data mapping requirements (See Fig. 6).

The three systems presented here were successfully implemented only after some trial and error and research to find the most appropriate tools for our needs. Although the individual tool may be simple to set up, there are many challenges to implementation that need to be overcome in order to establish a successful system that can be used by the stakeholders. Introducing new technology into an area that lacks complete infrastructure of electricity and roads requires preparation for a lack of access and power shortages. The introduction of technology must also be culturally appropriate, and training from a basic level is necessary especially for the older generation not accustomed to using mobile tools. In some cases, the cost of the mobile phone or netbook provided to an individual may be higher than what they can expect to earn in several months. As a result, the importance of the device and consequences for its loss must be established. It is possible to imagine that the importance of keeping such a device safe could prevent it being fully utilized.

Another barrier to the full acceptance and use of the new technology is simply the inertia against change. If people are used to filling out forms on paper, they may find it easier to continue with that system. They may not fully trust that data entered electronically is saved and accessible without a hard copy, and may end up doing double entry on paper forms in order to have physical proof of the work they have done. In the livestock disease surveillance project in Kisumu, the animal health technicians were determined to keep several sets of records of the cases they saw, both on paper and electronically. This was very useful as a back-up but may reduce the perceived advantage that mobile data collection reduces the time required for data entry. On the other hand, in the cases above, the end users of the technology were excited and proud to be entrusted with using a new device to assist with research.

Despite the challenges of implementation, mobile technology offers many advantages to animal health surveillance projects. In addition to improving speed and minimizing errors in data submission, these projects provide opportunities for building connections between veterinary research and information technology groups, and capacity building of both of these groups in developing countries. Providing access to and training in the use of mobile technologies is not just useful for one project, but will enable users to utilize mobile connections in other parts of their lives.

\section{ACKNOWLEDGEMENTS}

During the set-up of the SurveyToGo questionnaire, the assistance and guidance of the Dooblo team was highly appreciated and also the prompt reply to any of are queries.

\section{FINANCIAL SUPPORT}

The authors would like to thank the Belgian Science Policy Office (BELSPO) for their financial support of the Stereo II project entitled TickRisk (SR/00/144). Also the Belgian Development Cooperation (DGD) is thanked for their financial support of the FA3 programme. The syndromic livestock disease surveillance in Kenya was supported by the Wellcome Trust, UK (grant number 081828/B/06/Z) and the Compton Foundation, USA.

\section{REFERENCES}

Aanensen, D. M., Huntley, D. M., Feil, E. J., al-Own, F. and Spratt, B. G. (2009). EpiCollect: linking smartphones to web applications for epidemiology, ecology and community data collection. PLoS One 4(9), e6968. doi: 10.1371/journal.pone.0006968.

Anon (2005). Livestock Sector Brief. Kenya, Rome: FAO. http://www. fao.org/ag/againfo/resources/en/publications/sector_briefs/lsb_KEN.pdf (Accessed 11 December 2011).

Blycroft. (2008). http://www.africantelecomsnews.com (Accessed 13 December 2011).

DataDyne Group LLC (2011). Pricing. http://www.datadyne.org/ episurveyor/pricing (Accessed February 1, 2012).

Madder, M., Adehan, S., De Deken, R., Adehan, R. and Lokossou, R. (2012). New foci of Rhipicephalus microplus in West Africa. Experimental and Applied Acarology 56, 385-390. doi: 10.1007/s10493-012-9522-4.

Madder, M., Thys, E., Achi, L., Toure, A. and De Deken, R. (2011). Rhipicephalus (Boophilus) microplus: a most successful invasive tick species in West-Africa. Experimental and Applied Acarology 53, 139-145. doi:10.1007/s10493-010-9390-8.

Madder, M., Thys, E., Geysen, D., Baudoux, C. and Horak, I. (2007).

Boophilus microplus ticks found in West Africa. Experimental and Applied Acarology 43, 233-234. doi: 10.1007/s10493-007-9110-1.

MobileActive.org (2010). https://spreadsheets.google.com/pub?key= 0AgrRSBfpL7rXdGlEMzhBVXdmZ3o5bXdfLVZGVVU0YlE\&hl = en\&gid $=1$ (Accessed 25 January 2012).

OIE (2009). WAHID Interface. http://www.oie.int/wahis/public.php?page= home. (Accessed 25 January 2012).

OIE (2011). Terrestrial Animal Health Code Article 1.4.1. http://www.oie. $+=$ int/fileadmin/Home/eng/Health_standards/tahc/2010/en_chapitre_1.1.4. htm (Accessed 13 December 2011).

Qekwana, N.D., McCrindle, C. M.E. and Masipa, A. (2010). Rapid spatial and temporal outbreak investigations using cell phone technology: a pilot study. Paper presented at the Proceedings of the 9th annual congress of the Southern African Society For Veterinary Epidemiology and Preventive Medicine.

Robertson, C., Sawford, K., Daniel, S. L., Nelson, T.A. and Stephen, C. (2010). Mobile phone-based infectious disease surveillance system, Sri Lanka. Emerging Infectious Diseases 16, 1524-1531. doi: 10.3201/ eid1610.100249.

Schuster, C. and Brito, C.P. (2011). Cutting costs, boosting quality and collecting data real-time-Lessons from a cell phone-based beneficiary survey to strengthen Guatemala's Conditional Cash Transfer ProgramEnbreve, 166. http://www.worldbank.org/enbreve (Accessed 13/ 12/2011).

Shirima, K., Mukasa, O., Schellenberg, J. A., Manzi, F., John, D. Mushi, A., Mrisho, M., Tanner, M., Mshinda, H. and Schellenberg, D. (2007). The use of personal digital assistants for data entry at the point of collection in a large household survey in southern Tanzania. Emerging Themes in Epidemiology 4, 5. doi: 10.1186/ 1742-7622-4-5.

World Bank (2008). African Development Indicators (ADI), World Bank. Yang, C. H., Yang, J., Luo, X. S. and Gong, P. (2009). Use of mobile phones in an emergency reporting system for infectious disease surveillance after the Sichuan earthquake in China. Bulletin of the World Health Organization 87, 619-623. doi: 10.2471/BLT.08.060905. 